\title{
ANALYSIS OF A TWO-SCALE CAHN-HILLIARD MODEL FOR BINARY IMAGE INPAINTING
}

\author{
ANDREA BERTOZZI $^{1}$, SELIM ESEDOḠLU ${ }^{2}$, AND ALAN GILLETTE ${ }^{1}$
}

\begin{abstract}
Image inpainting is the process of filling in missing parts of damaged images based on information gleaned from surrounding areas. We consider a model for inpainting binary images using a modified Cahn-Hilliard equation. We prove for the steady state problem that the isophote directions are matched at the boundary of inpainting regions. Our model has two scales, the diffuse interface scale, $\varepsilon$, on which it can accomplish topological transitions, and the feature scale of the image. We show via simulations that a dynamic two step method involving the diffuse interface scale allows us to connect regions across larger inpainting domains. For the model problem of stripe inpainting, we show that this issue is related to a bifurcation structure with respect to the scale $\varepsilon$.
\end{abstract}

Key words. Cahn-Hilliard equation, image inpainting, binary images, fast numerical technique

1. Introduction. Image inpainting is the process of filling in missing parts of damaged images based on information gleaned from the surrounding areas. It is essentially a type of interpolation. Its applications include restoration of old paintings by museum artists [12], removing scratches from old photographs [7], altering scenes in photographs [20], and restoration of motion pictures [21]. Inpainting is intimately connected with other well-known computer vision problems, such as disocclusion and segmentation with depth. Indeed, some of the earlier work in these areas, particularly the work of Nitzberg, Mumford, and Shiota [26] on segmentation with depth, and the work of Masnou and Morel [24] on disocclusion, have provided motivation for a number of inpainting algorithms.

The work of Bertalmio et. al. [5] introduced image inpainting as a new research area of digital image processing. Their model is based on nonlinear partial differential equations, and is designed to imitate the techniques of museum artists who specialize in restoration. In particular, Bertalmio et. al. elucidated the principle that good inpainting algorithms should propagate sharp edges in surrounding areas into the damaged parts that need to be filled in. This can be done for instance by connecting contours of constant greyscale image intensity (called isophotes) to each other across the inpainting region, so that grey levels at the edge of the the damaged region get extended to the interior continuously. They also impose the direction of isophotes as a boundary condition at the edge of the inpainting domain.

In subsequent work with Bertozzi [4], they realized that the nonlinear PDE introduced in [5] has intimate connections to the two-dimensional fluid dynamics of the Navier-Stokes equation. Indeed, it turns out that the steady state problem originally proposed in [5] is equivalent to the inviscid Euler equations from incompressible flow, in which the image intensity function plays the role of the stream function in the fluid problem. This analogy also shows why diffusion is required in the original inpainting problem. The natural boundary conditions for inpainting are to match the

${ }^{*}$ May 14, 2007. ${ }^{1}$ Department of Mathematics, University of California Los Angeles and ${ }^{2}$ Department of Mathematics, University of Michigan, Ann Arbor, bertozzi@math.ucla.edu, esedoglu@umich.edu, alan.gillette@yahoo.com. This work was supported by the National Science Foundation and the intelligence community through the joint "Approaches to Combat Terrorism" program (NSF Grant AST-0442037). This work is also supported by ONR grant N000140410078 and NSF grants CCF-0321917 and DMS-0410085. 
image intensity on the boundary of the inpainting region and also the direction of the isophote lines, which for the fluid problem is effectively a generalized 'no-slip' boundary condition that requires a Navier-Stokes formulation, introducing a diffusion term. In practice nonlinear diffusion (as in Perona-Malik [29], and Rudin, Osher, Fatemi [31]) works very well to avoid blurring of edges in the inpainting.

A different approach to inpainting was proposed by Chan and Shen in one of the earliest works [9] in the field. They introduced the idea that well-known variational image denoising and segmentation models can be easily adapted to the inpainting task by a simple modification. Indeed, these models often include a fidelity term that keeps the solutions close to the given image. By restricting the effects of the fidelity term in these models to only the complement of the inpainting region, Chan and Shen showed that very good image completions can be obtained. The principle behind their approach can be summarized as follows: Variational denoising and segmentation models all have an underlying notion of what constitutes an image. In the inpainting region, the models of Chan and Shen reconstruct the missing image features by relying on this built in notion of what constitutes a natural image.

The first model introduced by Chan and Shen used the total variation based image denoising model of Rudin, Osher, and Fatemi [31] for the inpainting purpose. This model can successfully propagate sharp edges into the damaged domain. However, because the regularization term in this model exacts a penalty on the length of edges, the inpainting model cannot connect contours across very large distances. Another caveat is that this model does not keep the direction of isophotes continuous across the boundary of the inpainting domain.

Subsequently, Kang, Chan, and Shen [8] introduced a new variational image inpainting model that addressed the caveats of the the total variation based one. This model is motivated by the work of Nitzberg, Mumford, and Shiota [26], and includes a new regularization term that penalizes not merely the length of edges in an image, but the integral of the square of curvature along the edge contours. This allows both for isophotes to be connected across large distances, and their directions to be kept continuous across the edge of the inpainting region. Another source of motivation was the work of Masnou and Morel [23, 24], where a formal variational formulation for disocclusion and a particular practical implementation is given: they use geodesic curves to join the isophotes arriving at the boundary of the inpainting region.

Following in the footsteps of Chan and Shen, Esedoglu and Shen [14] adapted the Mumford-Shah image segmentation model to the inpainting problem. They utilized Ambrosio and Tortorelli's elliptic approximations [2] to the Mumford-Shah functional. Gradient descent for these approximations leads to parabolic equations with a small parameter $\varepsilon$ in them; they represent edges in the image by transition regions of thickness $\varepsilon$. These equations have the benefit that highest order derivatives are linear. They can therefore be solved rather quickly. However, like the total variation image denoising model, the Mumford-Shah segmentation model penalizes length of edge contours, and therefore does not allow for the connection of isophotes across large distances in inpainting applications.

In order to improve the utility of the Mumford-Shah model in inpainting, Esedoglu and Shen introduced the Mumford-Shah-Euler image model that, just like the previous work [8] of Kang, Chan, Shen, penalizes square of the curvature along an edge contour. Following previous work by March [22], they then used a conjecture of De Giorgi [18] (which has since been given a proof in [30]) to approximate the resulting variational 
problem by elliptic ones. The same approximation is also used in [13] to compute piecewise constant segmentations with depth via the model of Nitzberg, Mumford, and Shiota. Resulting gradient descent equations are fourth order, nonlinear parabolic PDE with a small parameter in them, and have a striking resemblance to the CahnHilliard equation.

A recent paper by the authors [6] presents a new model directly based on CahnHilliard evolution, rather than a more complex gradient flow to minimize a curvature functional. This simpler model was shown, in numerical examples, to have many of the desirable properties of the model introduced in [14]. In particular, both image intensity and the direction of edges are continued smoothly across the inpainting region. However the Cahn-Hilliard method was shown to be 2-5 times faster, computationally, than that of [14] and an order of magnitude or more faster than other competing PDE-based inpainting methods. In this paper, we present rigorous theory for the existence of solutions of the evolution equation. We also prove, for the steady state problem, that in the limit of large fidelity, isophote directions of the restored image will match those of the given background image, provided it has sufficient regularity. Section 2 provides a review of previous phase field models for image processing, in which a small scale $\varepsilon$ is introduced over which boundaries of objects are diffused. Section 3 reviews the proposed model. Section 4 presents rigorous existence theory for the time evolving problem. Section 5 proves continuity of isophotes for the case $\varepsilon=1$ and a background image function that is at least $C^{2}$. Section 6 considers binary data $f$, and shows that for sufficiently small $\varepsilon$, the direction of edges of the restored image $u$ again match the direction of edges of the binary data $f$ in the limit of large fidelity. As in Section 5, this is accomplished by looking at the large fidelity limit. Section 7 considers a model problem of stripe inpainting. This problem is particular useful to understand the existence of multiple stable solutions of the inpainting problem and how they are related to each other through a bifurcation analysis. Section 8 presents some real world computational examples with binary data. Section 9 presents a discussion with suggestions for future work.

2. Phase Field Models for Inpainting. We recall very briefly the two models introduced in [14], and their approximations by elliptic functionals. The first one is a very simple modification of the Mumford-Shah segmentation model, and has the following form: For a given image $f(x)$, solve the minimization problem

$$
\inf _{\substack{u(x) \in L^{2}(\Omega) \\ K \subset \Omega}} \int_{\Omega \backslash K}|\nabla u|^{2} d x+\alpha \operatorname{Length}(K)+\lambda \int_{\Omega \backslash D}(f-u)^{2} d x .
$$

Here, the unknown set $K$ is supposed to be a union of curves and approximate the edges of the given image $f(x)$. The function $u(x)$, which is also an unknown of the problem, is required to be smooth away from the edge set $K$ by the Dirichlet energy that appears in the energy. $D \subset \Omega$ is the user supplied inpainting region. The last integral in (2.1) represents the fidelity term, and forces the piecewise smooth function $u(x)$ to remain close in the $L^{2}$ sense to the given image $f(x)$. The only difference of (2.1) from the original Mumford-Shah functional is that the fidelity term is integrated over $\Omega \backslash D$ instead of the entire domain $\Omega$.

Energies of the form (2.1) are difficult to handle because part of the minimization is to be carried out over collections of curves in the plane. Ambrosio and Tortorelli introduced elliptic energies that approximate the Mumford-Shah functional in the sense of Gamma convergence (details can be found in [10]), whose numerical treatments are 
consequently much easier. Their approximation, when written for (2.1), takes the form:

$$
M S_{\varepsilon}(u, z)=\int_{\Omega}\left(z^{2}|\nabla u|^{2}+\alpha\left(\varepsilon|\nabla z|^{2}+\frac{(1-z)^{2}}{4 \varepsilon}\right)\right) d x+\lambda \int_{\Omega \backslash D}(f-u)^{2} d x .
$$

Here, the function $z$ is introduced to keep track of the edge set. As the small parameter $\varepsilon \rightarrow 0$, these energies have been rigorously proved to converge to (2.1) in the sense of Gamma convergence. The implication is that any accumulation point of minimizers of (2.2) has to be a minimizer of (2.1). These approximations are often called 'diffuse interface' approximations because for a fixed value of $\varepsilon$, the minimizer approximates the sharp interface problem by one in which there is an interface of thickness of order $\varepsilon$. Diffuse interface methods are particular useful for problems in which topology transitions of the interface are of interest.

Esedoğlu and Shen [14] introduce a variant of energy (2.1) that incorporates curvature of edge contours into the functional. It has the form:

$$
M S E(u, K)=\int_{\Omega \backslash K}|\nabla u|^{2} d x+\int_{K}\left(\alpha+\beta \kappa^{2}\right) d \sigma+\lambda \int_{\Omega \backslash D}(u-f)^{2} d x .
$$

where $\kappa$ is the curvature of $K$. Based on a conjecture of E. De Giorgi [18], and following previous work by March in [22], they consider the following diffuse interface approximation of $(2.3)$ :

$$
\begin{aligned}
M S E_{\varepsilon}(u, z)=\int_{\Omega}\left(z^{2}|\nabla u|^{2}\right. & +\alpha\left[\varepsilon|\nabla z|^{2}+\frac{1}{\varepsilon} W(z)\right] \\
& \left.+\frac{\beta}{\varepsilon}\left[2 \varepsilon \Delta z-\frac{1}{\varepsilon} W^{\prime}(z)\right]^{2}\right) d x+\lambda \int_{\Omega \backslash D}(u-f)^{2} d x .
\end{aligned}
$$

where $W(z)$ is the double-welled function $W(z)=z^{2}(z-1)^{2}$. Gradient descent for (2.4) with respect to the $L^{2}$ inner product gives a system of coupled diffusion equations. The addition of curvature terms introduced in (2.3) allow for control of edge directions as well as location of edges. This naturally leads to a higher order energy in the diffuse interface approximation (2.4). The result is an $L^{2}$ gradient flow that is fourth order in space and includes terms that scale as $1 / \epsilon^{3}$. In the next section we show that a much simpler model based on the original length functional of (2.1) can control both edge locations and directions, provided that we consider a superposition of an $H^{-1}$ gradient flow and $L^{2}$ flow, rather than a pure $L^{2}$ descent.

3. Proposed Model. In this paper, we consider a binary image (i.e shape) inpainting model that is a much simplified version of the Esedog $l u-S h e n$ model from the previous section. The key observation leading to the model is that the fourth order gradient flow in the Esedoḡlu-Shen model, has features in common with the CahnHilliard equation, which is a much simpler model for which fast solution techniques are available $[6,37]$. It is therefore natural to ask if a simpler model can be used directly for inpainting.

Let $f(x)$ be a given binary image, and suppose that $D \subset \Omega$ is the inpainting domain. We propose solving the following equation to steady state in order to construct an inpainted version $u(x)$ of $f(x)$ :

$$
u_{t}=-\Delta\left(\varepsilon \Delta u-\frac{1}{\varepsilon} W^{\prime}(u)\right)+\lambda(x)(f-u) .
$$


where

$$
\lambda(x)=\left\{\begin{array}{cl}
0 & \text { if } x \in D \\
\lambda_{0} & \text { if } x \in \Omega \backslash D
\end{array}\right.
$$

$u(x, t)$ satisfies $\frac{\partial u}{\partial \nu}=\frac{\partial \Delta u}{\partial \nu}=0$ on $\partial \Omega$, and again $W(u)=u^{2}(u-1)^{2}$.

Equation (3.1) is identical with the standard Cahn-Hilliard equation [3, 27] except for the second term on the right hand side. This term is there to keep the solution constructed close to the given image $f(x)$ in the complement of the inpainting domain, where there is image information available. We mention here that such phase field models have been used for other applications such as shape recovery in computer vision [11].

Equation (3.1) is not a gradient flow for an energy; however, it can be thought of as a superposition of gradient descents for two different energies. Indeed, the CahnHilliard equation is the gradient flow with respect to the $\mathrm{H}^{-1}$ inner product [35] of the following energy, which appeared already as a part of some of the variational models introduced in the previous sections:

$$
E_{\varepsilon}(u):=\int_{\Omega}\left(\frac{\varepsilon}{2}|\nabla u|^{2}+\frac{1}{\varepsilon} W(u)\right) d x .
$$

This is the energy of Modica-Mortola [25], which has been rigorously shown to approximate the perimeter of sets in the sense of Gamma convergence:

$$
E_{\varepsilon} \stackrel{\Gamma}{\longrightarrow} E(u):=\left\{\begin{array}{cl}
\operatorname{Per}(\Sigma) & \text { if } u(x)=\mathbf{1}_{\Sigma}(x) \text { for some } \Sigma \subset \Omega \\
+\infty & \text { otherwise }
\end{array}\right.
$$

When $\lambda(x) \equiv 0$, equation (3.1) thus decreases (3.2); it can also be easily seen that in this case the solution preserves total image intensity (i.e. $\int_{\Omega} u(x, t) d x$ is constant in t). The dynamics of (3.1) in this case has been studied extensively. For instance, it is well-known that under (3.1) with $\lambda(x) \equiv 0$, arbitrary initial data form interfaces of thickness approximately $\varepsilon$ at a fast time scale; these interfaces separate regions where the solution is approximately either 0 or 1 (location of wells for the potential $W)$. The fact that energy (3.2) is decreased suggests that the subsequent evolution involves some sort of coarsening of this configuration of regions. Indeed, as $\varepsilon \rightarrow 0$, at a slower time scale the interfaces approximate the solution of the Mullins-Sekerka problem $[1,28]$.

When $\lambda(x) \not \equiv 0$, equation (3.1) is no longer gradient descent for (3.2); the second term in the right hand side of (3.1) is gradient descent with respect to the $L^{2}$ inner product for the pointwise energy:

$$
\int_{\Omega}(u-f)^{2} d x
$$

Our proposed model (3.1) can thus be thought of as a superposition of gradient descent with respect to $H^{-1}$ inner product for (3.2), and gradient descent with respect to $L^{2}$ inner-product for (3.3). However, it is not the gradient descent, either in $H^{-1}$ or $L^{2}$ inner product, for the sum of the energies (3.2) and (3.3).

An important distinction of model (3.1) from those of Bertalmio et. al. is that no explicit boundary conditions are imposed at the boundary $\partial D$ of the inpainting region 
$D$. However, we will show in Section 5 that in the limit that $\lambda_{0} \rightarrow \infty$, stationary solutions of (3.1) converge to the solution of the following equation:

$$
\begin{aligned}
& -\Delta\left(\varepsilon \Delta u-\frac{1}{\varepsilon} W^{\prime}(u)\right)=0 \text { in } x \in D, \\
& u(x)=f(x) \text { on } x \in \partial D, \text { and } \\
& \nabla u=\nabla f \text { on } x \in \partial D .
\end{aligned}
$$

This is proved under the condition that the given function $f(x) \in C^{2}(\Omega)$. The fact that (3.1) is fourth order naturally leads to the two boundary conditions (3.4) in the limit of large $\lambda$. We note that this feature is not special to the particular nonlinear equation considered here, but is due to the highest order term on the right hand side of (3.1). The rigorous results of sections 5 and 6 require only the highest order term to complete the analysis. The rest of the work in those sections is to show that the lower order terms, which are responsible for the phase separation, do not adversely affect the results. We therefore expect that solving equation (3.1) with a very large choice of the constant $\lambda_{0}$ will approximate a solution of (3.4).

After addressing the case $\lambda \rightarrow \infty$ for $f \in C^{2}$, where we take $\epsilon$ first to be one, we then consider $f$ a smooth approximation of a binary function, where the smoothing is on a scale $\epsilon$, the diffuse interface scale. We show that the same estimates hold as in the completely smooth case, except that now $\lambda$ depends on $\epsilon$. In practice, we do not find any significant numerical hardship regarding the large value of $\lambda$ when $\epsilon$ is small.

From the inpainting application point of view, the ability to impose boundary conditions for both the solution $u(x)$ and its derivative $\nabla u$ is one of the great advantages of fourth order models such as (3.4). Indeed, this allows image information generated by the model in the inpainting region $D$ to match the original image data defined on $\Omega \backslash D$ not only in intensity, but also in isophote directions. That means our model (3.1) continues edges into the inpainting domain without introducing kinks at the boundary $\partial D$.

4. Global existence of weak solutions of the modified Cahn-Hilliard equation. Before discussing the steady state problem, we show that well-posedness of the dynamic problem follows from classical methods for the case $\lambda_{0}=0$.

Consider the time dependent problem on a compact region $\Omega \subset \mathbb{R}^{2}$ with an inpainting region $D \subset \Omega$,

$$
u_{t}=-\Delta\left(\varepsilon \Delta u-\frac{1}{\varepsilon} W^{\prime}(u)\right)+\lambda_{0}(f-u) \chi_{\Omega \backslash D} .
$$

Following III, 4.2 in [36] for the case $\lambda_{0}=0$, we define

$$
V=\left\{\phi \in H^{2}(\Omega) \mid \frac{\partial \phi}{\partial \nu}=0 \quad \text { on } \quad \partial \Omega\right\}
$$

We define a weak solution of the evolution equation (4.1) as one that satisfies

$$
\frac{d}{d t}\langle u, v\rangle+\langle\varepsilon \Delta u, \Delta v\rangle-\left\langle\frac{1}{\varepsilon} W^{\prime}(u), \Delta v\right\rangle=\langle\lambda(x)(f-u), v\rangle, \quad \forall v \in V,
$$

where $\langle.,$.$\rangle specifies the L^{2}$ inner product.

We establish the following global existence and uniqueness theorem. 
THEOREM 4.1. For every $u_{0}$ in $L^{2}(\Omega)$, and every $T>0$, the initial-boundary value problem (4.2) has a unique solution $u$ which belongs to $C\left([0, T] ; L^{2}(\Omega)\right) \cap L^{2}(0, T ; V)$.

The proof of existence follows a similar argument as in [36] for $\lambda=0$. We require an $L^{2}$ estimate that includes the additional fidelity term. In fact we show that this gives a global in time bound for $u$ in $L^{2}$ when $\lambda$ is sufficiently large.

LEMma 4.2. Given a weak solution as described above, there exist constants $C(\varepsilon, \lambda, f)>0$ and $\theta(\lambda, f, \varepsilon)$ so that

$$
\frac{1}{2} \frac{d}{d t} \int_{\Omega} u^{2} d x \leq C(\varepsilon, \lambda, f)-\theta \int_{\Omega / D} u^{2} d x
$$

for all $t \geq 0$. For $\lambda$ sufficiently large, $\theta>0$. The constant $C$ also depends on the size of the domain $D$.

This lemma establishes an a priori bound for the $L^{2}$ norm of the solution $u$; this bound is uniform in time for $\lambda$ sufficiently large. We expect that it would therefore play an important role in, for example, establishing existence of steady states for the modified Cahn-Hilliard equation considered in this paper.

Proof: We first reference a standard interpolation inequality:

$$
\int_{\Omega}|\nabla u|^{2} d x \leq \delta \int_{\Omega}(\Delta u)^{2} d x+\frac{C}{\delta} \int_{\Omega} u^{2} d x
$$

By the $L^{1}$ version of Poincare's inequality, together with the observation that the domain of integration in the second integral of equation (4.4) can be taken to be something smaller than $\Omega$ (at the expense of larger constants, but no matter):

$$
\int_{\Omega} u^{2} d x \leq C \int_{\Omega}\left|\nabla\left(u^{2}\right)\right| d x+C \int_{\Omega \backslash D} u^{2} d x
$$

where $C$ depends on the size of $D$ compared to $\Omega$. By Hölder's inequality we also have that (for some $\alpha$ small enough):

$$
\int_{\Omega}\left|\nabla\left(u^{2}\right)\right| d x \leq|\Omega|^{\frac{1}{2}}\left(\int_{\Omega} u^{2}|\nabla u|^{2} d x\right)^{\frac{1}{2}} \leq \frac{\alpha}{2} \int_{\Omega} u^{2}|\nabla u|^{2} d x+\frac{C}{2 \alpha}
$$

Putting the last three inequalities together:

$$
\int_{\Omega}|\nabla u|^{2} d x \leq \delta \int_{\Omega}(\Delta u)^{2} d x+\frac{C \alpha}{2 \delta} \int_{\Omega} u^{2}|\nabla u|^{2} d x+\frac{C}{\delta} \int_{\Omega \backslash D} u^{2} d x+\frac{C}{2 \alpha \delta}
$$

Now computing the rate of change of the $L^{2}$ norm of the solution, we get that:

$$
\begin{aligned}
\frac{1}{2} \frac{d}{d t} \int_{\Omega} u^{2} d x & =-\varepsilon \int_{\Omega}(\Delta u)^{2} d x+\frac{1}{\varepsilon} \int_{\Omega} u \Delta W^{\prime}(u) d x+\lambda \int_{\Omega \backslash D} u(f-u) d x \\
& =-\varepsilon \int_{\Omega}(\Delta u)^{2} d x-\frac{1}{\varepsilon} \int_{\Omega} W^{\prime \prime}(u)|\nabla u|^{2} d x+\lambda \int_{\Omega \backslash D} u(f-u) d x
\end{aligned}
$$

where we integrated by parts on the second term in the right hand side. 

(4.8):

Using the fact that $W^{\prime \prime}(\xi) \geq \gamma \xi^{2}-C$ for all $\xi$, for some constants $\gamma$ and $C$, in

$\frac{1}{2} \frac{d}{d t} \int_{\Omega} u^{2} d x \leq-\varepsilon \int_{\Omega}(\Delta u)^{2} d x-\frac{\gamma}{\varepsilon} \int_{\Omega} u^{2}|\nabla u|^{2} d x+\frac{C}{\varepsilon} \int_{\Omega}|\nabla u|^{2}+\lambda \int_{\Omega \backslash D} u(f-u) d x$

We now put everything together as follows: First, writing the last term above as

$$
\lambda \int_{\Omega / D} u f d x-\lambda \int_{\Omega / D} u^{2} d x \leq \frac{\lambda}{2} \int_{\Omega / D} f^{2} d x-\frac{\lambda}{2} \int_{\Omega / D} u^{2} d x
$$

we use inequality (4.5) in order to bound the last term of the inequality above as follows:

$$
\begin{aligned}
\frac{1}{2} \frac{d}{d t} \int_{\Omega} u^{2} d x \leq-\varepsilon \int_{\Omega}(\Delta u)^{2} d x & -\frac{\gamma}{\varepsilon} \int_{\Omega} u^{2}|\nabla u|^{2} d x+\frac{C}{\varepsilon} \int_{\Omega}|\nabla u|^{2} d x \\
& +\frac{\lambda}{2} \int_{\Omega \backslash D} f^{2} d x+\frac{\lambda}{2} \int_{\Omega}\left|\nabla\left(u^{2}\right)\right| d x-\frac{\lambda}{2 C} \int_{\Omega} u^{2} d x .
\end{aligned}
$$

Now use inequality (4.6) with $\alpha=\delta_{1}$ to estimate the next to last term in the inequality above:

$$
\begin{array}{r}
\frac{1}{2} \frac{d}{d t} \int_{\Omega} u^{2} d x \leq-\varepsilon \int_{\Omega}(\Delta u)^{2} d x-\frac{\gamma}{\varepsilon} \int_{\Omega} u^{2}|\nabla u|^{2} d x+\frac{C}{\varepsilon} \int_{\Omega}|\nabla u|^{2} d x+\frac{\lambda}{2} \int_{\Omega \backslash D} f^{2} d x \\
+\frac{\lambda \delta_{1}}{4} \int_{\Omega} u^{2}|\nabla u|^{2} d x+\frac{C \lambda}{4 \delta_{1}}-\frac{\lambda}{2 C} \int_{\Omega} u^{2} d x
\end{array}
$$

Now use inequality (4.7) with $\alpha=\delta_{1}$ and $\delta=\delta_{2}$ to estimate the $\int|\nabla u|^{2} d x$ term in the inequality above as follows:

$$
\begin{aligned}
& \frac{1}{2} \frac{d}{d t} \int_{\Omega} u^{2} d x \leq-\varepsilon \int_{\Omega}(\Delta u)^{2} d x-\frac{\gamma}{\varepsilon} \int_{\Omega} u^{2}|\nabla u|^{2} d x+\frac{C \delta_{2}}{\varepsilon} \int_{\Omega}(\Delta u)^{2} d x+\frac{C \delta_{1}}{2 \varepsilon \delta_{2}} \int_{\Omega} u^{2}|\nabla u|^{2} d x \\
+ & \frac{C}{2 \varepsilon \delta_{1} \delta_{2}}+\frac{C}{\varepsilon \delta_{2}} \int_{\Omega \backslash D} u^{2} d x+\frac{\lambda}{2} \int_{\Omega \backslash D} f^{2} d x+\frac{\lambda \delta_{1}}{4} \int_{\Omega} u^{2}|\nabla u|^{2} d x+\frac{C \lambda}{4 \delta_{1}}-\frac{\lambda}{2 C} \int_{\Omega} u^{2} d x .
\end{aligned}
$$

We now try to satisfy the following conditions with our choice of the constants $\delta_{1}, \delta_{2}$, and $\lambda$ :

$$
\begin{aligned}
& \text { 1. } \frac{C \delta_{2}}{\varepsilon}<\varepsilon \text {, i.e. } \delta_{2}<\frac{\varepsilon^{2}}{C}, \\
& \text { 2. } \frac{C \delta_{1}}{2 \varepsilon \delta_{2}}+\frac{\lambda \delta_{1}}{4}<\frac{\gamma}{\varepsilon}, \\
& \text { 3. } \frac{C}{\varepsilon \delta_{2}}<\frac{\lambda}{2 C},
\end{aligned}
$$

To satisfy the first condition, take $\delta_{2}=\frac{1}{8 C} \varepsilon^{2}$. Then, to satisfy the third, we can choose any $\lambda \geq \frac{16 C^{3}}{\varepsilon^{3}}$. To satisfy the second, choose $\delta_{1}<\frac{4 \varepsilon^{2} \gamma}{16 C^{2}+\lambda \varepsilon^{3}}$. With these choices, we end up with the following inequality:

$$
\frac{1}{2} \frac{d}{d t} \int_{\Omega} u^{2} d x \leq C(\varepsilon, \lambda, f)-\theta \int_{\Omega} u^{2} d x
$$

where $\theta>0$. For any $\lambda$, Grönwall's lemma implies an a priori bound on the $L^{2}$ norm of $u$ on any finite time interval $[0, T)$. Moreover, for sufficiently large $\lambda$, we obtain a uniform in time bound on the $L^{2}$ norm of $u(\cdot, t)$ :

$$
\exists M>0 \text { such that }\|u(\cdot, t)\|_{L^{2}} \leq M \text { for all } t \geq 0 .
$$


Remark: In the above analysis, the $\lambda$ needed to obtain a negative $\theta$ depends on $\epsilon$ and the size of the inpainting region compared to $\Omega$.

Following the remaining arguments in [36] one can establish global existence and uniqueness of a weak solution to the modified Cahn-Hilliard equation. We are not aware of any Lyapunov function for this problem, as in the original $\mathrm{CH}$ model. However, we observe in our numerical simulations that the solution quickly approaches a steady state as $t$ increases; as we mentioned before, the existence of such are strongly suggested by the estimate given above. Moreover, the steady state solution appears to inherit the regularity of the original parabolic problem. In the next section we show that existence of an $H^{2}$ solution of the steady state problem guarantees that the intended boundary conditions for the inpainting problem are satisfied as $\lambda \rightarrow \infty$. In the analysis, $\lambda$ depends on $\epsilon$, however this dependence is not something that, in practice, causes us hardship in the computation. We prove this result while noting that convergence of the time dependent solution to the steady state problem remains unaddressed.

5. Fidelity and boundary conditions. The fidelity parameter $\lambda$ in (3.1) enforces the original image outside of the inpainting region. One might expect that as $\lambda$ gets large, the existing region enforces some kind of effective boundary conditions on the inpainting region. In this section we prove this rigorously for the steady state problem. As we mentioned earlier, these solutions turn out to approximate a solution of (3.4). Our results establish rigorously a connection between the inpainting technique used by Bertalmio et. al. (who prefer to impose boundary conditions at the edge of the inpainting domain $D$ ) and that of Chan et. al. (who prefer to use a fidelity term, similar to the second term in the right hand side of our model (3.1)). In this section we consider the case $\varepsilon=1$, and smooth (greyscale) $f$. In the next section we show how to extend these results to binary $f$ and small $\varepsilon$.

5.1. Key estimates. We require the following version of the Poincaré inequality.

Lemma 5.1. (Poincaré inequality) Let $\Omega \subset \mathbb{R}^{N}$ be a bounded domain with Lipschitz boundary. There exists a constant $C=C(\Omega)>0$ such that if $v(x) \in C^{1}(\Omega)$ with $v=(u-\bar{u})^{2}$ for some $u \in C^{1}(\Omega)$ and $\bar{u}:=\frac{1}{|\Omega|} \int_{\Omega} u d x$, then

$$
\int_{\Omega} v^{2} d x \leq C \int_{\Omega}|\nabla v|^{2} d x
$$

Proof: Suppose not. Then there exists a sequence $\left\{u_{j}\right\}_{j=1}^{\infty} \subset C^{1}(\Omega)$ such that

$$
\int_{\Omega} v_{j}^{2} d x>j \int_{\Omega}\left|\nabla v_{j}\right|^{2} d x
$$

where $v_{j}=\left(u_{j}-\bar{u}_{j}\right)^{2}$ for some $u_{j} \in C^{1}(\Omega)$, and $v_{j} \neq 0$. By normalizing, we make sure that

$$
\int_{\Omega} v_{j}^{2} d x=1 \text { and } \bar{u}_{j}:=\frac{1}{|\Omega|} \int_{\Omega} u_{j} d x=0 \text { for all } j .
$$

Then, the functions $v_{j}$ are bounded uniformly in $L^{2}(\Omega)$ and

$$
\int_{\Omega}\left|\nabla v_{j}\right|^{2} d x<\frac{1}{j}
$$


By Rellich's theorem, by passing to a subsequence if necessary, we may assume that $v_{j}$ converge to some $v_{\infty}$ in the $L^{2}(\Omega)$ sense and pointwise a.e.

Let $w_{j}(x):=\max ^{2}\left\{0, u_{j}(x)\right\}$. Then:

$$
\begin{aligned}
\int_{\Omega}\left|\nabla w_{j}\right|^{2} d x & \leq \int_{\Omega} 4\left(\max \left\{0, u_{j}(x)\right\}\right)^{2}\left|\nabla u_{j}\right|^{2} d x \leq \int_{\Omega} 4 u_{j}^{2}\left|\nabla u_{j}\right|^{2} d x \\
& =4 \int_{\Omega}\left|\nabla v_{j}\right|^{2} d x \longrightarrow 0 \text { as } j \rightarrow \infty
\end{aligned}
$$

Once again by Rellich, we may assume that the sequence $w_{j}$ converges to some $w_{\infty}$ in $L^{2}(\Omega)$. By lower semicontinuity,

$$
\int_{\Omega}\left|\nabla w_{\infty}\right|^{2} d x \leq \liminf _{j \rightarrow \infty} \int_{\Omega}\left|\nabla w_{j}\right|^{2} d x=0
$$

That means $w_{\infty}$ is a constant.

The very same argument applied to $\tilde{w}_{j}(x):=\min ^{2}\left\{0, u_{j}(x)\right\}$ shows that up to passing to subsequences, we may assume that $\tilde{w}_{j} \rightarrow \tilde{w}_{\infty}$ in $L^{2}(\Omega)$ and pointwise a.e., where $\tilde{w}_{\infty}$ is a constant.

Thus, for a.e. $x \in \Omega$, we have that $\sqrt{w_{j}(x)}=\max \left\{0, u_{j}(x)\right\}$ converges to $\sqrt{w_{\infty}}$ and $\sqrt{\tilde{w}_{j}(x)}=-\min \left\{0, u_{j}(x)\right\}$ converges to $\sqrt{\tilde{w}_{\infty}}$. Since $u_{j}(x)=\max \left\{0, u_{j}(x)\right\}+$ $\min \left\{0, u_{j}(x)\right\}$, we have that

$$
\lim _{j \rightarrow \infty} u_{j}(x)=C:=\sqrt{w_{\infty}}-\sqrt{\tilde{w}_{\infty}} \text { for a.e. } x \in \Omega .
$$

Moreover, since the sequence $\left\{a v_{j}^{2}+b\right\}$ dominates $\left\{\left(u_{j}-C\right)^{4}\right\}$ for some $a, b \in \mathbb{R}$, and $\left\{v_{j}\right\}$ converges in $L^{2}$, we get that the sequence $\left\{u_{j}\right\}$ converges to the constant $C$ in $L^{4}(\Omega)$. But $\bar{u}_{j}=0$, so in fact we must have $C=0$. This contradicts the fact $\int u_{j}^{4} d x=\int v_{j}^{2} d x=1$.

Lemma 5.2. Let $\Omega \subset \mathbb{R}^{N}$ be a bounded domain with Lipschitz boundary. Let $D$ be a compactly included subdomain of $\Omega$, also with Lipschitz boundary. There exist constants $C_{1}, C_{2}$, and $C_{3}$ such that if $u(x) \in C^{1}(\Omega)$, then

$$
\begin{aligned}
\int_{\Omega} u^{4} d x \leq & C_{1}\left(\frac{1}{|\Omega \backslash D|} \int_{\Omega \backslash D} u d x\right)^{4}+C_{2} \int_{\Omega} u^{2}|\nabla u|^{2} d x \\
& +C_{3}\left(\frac{1}{|\Omega \backslash D|} \int_{\Omega \backslash D} u d x\right)^{2} \int_{\Omega}|\nabla u|^{2} d x
\end{aligned}
$$

Proof: Define the function $v$ to be:

$$
v(x):=\left(u(x)-\frac{1}{|\Omega \backslash D|} \int_{\Omega \backslash D} u(x) d x\right)^{2} .
$$

The standard Poincaré inequality implies:

$$
\int_{\Omega} v^{2} d x \leq C \int_{\Omega \backslash D} v^{2} d x+C \int_{\Omega}|\nabla v|^{2} d x .
$$


On the other hand, Lemma 5.1 implies:

$$
\int_{\Omega \backslash D} v^{2} d x \leq C \int_{\Omega \backslash D}|\nabla v|^{2} d x
$$

Combining inequalities (5.1) and (5.2) we get:

$$
\int_{\Omega} v^{2} d x \leq C \int_{\Omega}|\nabla v|^{2} d x .
$$

Writing the last inequality in terms of $u$ yields the conclusion of the present lemma after a few elementary manipulations.

Proposition 5.3. Let $u$ be an $H^{2}$ weak solution of the PDE

$$
-\Delta\left(\Delta u-W^{\prime}(u)\right)+\lambda(x)(f-u)=0,
$$

that is

$$
\langle\Delta u, \Delta v\rangle-\left\langle W^{\prime}(u, \Delta v\rangle=\langle\lambda(x)(f-u), v\rangle, \quad \forall v \in V\right.
$$

where

$$
\lambda(x)=\left\{\begin{array}{cl}
0 & \text { if } x \in D, \\
\lambda_{0} & \text { if } x \in \Omega \backslash D
\end{array}\right.
$$

with $\lambda_{0} \geq 0$. Assume that $f \in C^{2}(\Omega)$. Then there exists constants $C_{1}$ and $C_{2}$ independent of $\lambda_{0}$, depending only on $f$, so that

$$
\begin{aligned}
& \int_{\Omega}(\Delta u)^{2} d x \leq C_{1}, \text { and } \\
& \int_{\Omega \backslash D}(u-f)^{2} d x \leq \frac{C_{2}}{\lambda_{0}} .
\end{aligned}
$$

Proof: First, we consider a test function $v \equiv 1$ to obtain

$$
\int_{\Omega \backslash D} u d x=\int_{\Omega \backslash D} f d x .
$$

where we used the fact $\lambda=0$ in $D$. Then, taking a test function $v=(u-f)$, we get

$$
\begin{aligned}
0 & =\int_{\Omega}-\left((\Delta u-\Delta f)\left(\Delta u-W^{\prime}(u)\right)+\lambda(x)(u-f)^{2}\right) d x \\
& =-\int_{\Omega}(\Delta u)^{2} d x-\int_{\Omega} \lambda(x)(f-u)^{2} d x+(I)+(I I)
\end{aligned}
$$

where

$$
\begin{aligned}
(I) & :=\int_{\Omega} W^{\prime}(u) \Delta u d x, \text { and } \\
(I I) & :=\int_{\Omega}\left(\Delta u-W^{\prime}(u)\right) \Delta f d x .
\end{aligned}
$$


By first applying integration by parts we have

$$
\begin{aligned}
(I) & =-\int_{\Omega} W^{\prime \prime}(u)|\nabla u|^{2} d x \\
& \leq \int_{\Omega}-\gamma u^{2}|\nabla u|^{2} d x+\int_{\Omega} C|\nabla u|^{2} d x .
\end{aligned}
$$

where we used the fact that there exist positive constants $\gamma$ and $C$ such that $W^{\prime \prime}(\xi) \geq$ $\gamma \xi^{2}-C$ for all $\xi \in \mathbb{R}$. By (5.5), Lemma 5.2 applied to $u$ gives:

$$
\int_{\Omega} u^{2}|\nabla u|^{2} d x \geq C \int_{\Omega} u^{4} d x-C \int_{\Omega}|\nabla u|^{2} d x-C .
$$

Hence,

$$
(I) \leq-C \gamma \int_{\Omega} u^{4} d x+C \gamma \int_{\Omega}|\nabla u|^{2} d x+C \gamma
$$

By Hölder's inequality,

$$
\int_{\Omega} u^{4} d x \geq C\left(\int_{\Omega} u^{2} d x\right)^{2} .
$$

Combining (5.7) and (5.8) and absorbing $\gamma$ into the constant $C$, we get

$$
(I) \leq-C \int_{\Omega} u^{4} d x-C\left(\int_{\Omega} u^{2} d x\right)^{2}+C \int_{\Omega}|\nabla u|^{2}+C .
$$

We now use the following standard interpolation inequality

$$
\int_{\Omega}|\nabla u|^{2} d x \leq \delta \int_{\Omega}(\Delta u)^{2} d x+C(\delta) \int_{\Omega} u^{2} d x .
$$

(where $\delta>0$ but arbitrarily small) along with (5.9) to obtain the following estimate:

$$
(I) \leq \delta \int_{\Omega}(\Delta u)^{2} d x-C \int_{\Omega} u^{4} d x-C\left(\int_{\Omega} u^{2} d x\right)^{2}+C(\delta) \int_{\Omega} u^{2} d x+C .
$$

We turn to estimating $(I I)$. Since $f \in C^{2}(\Omega)$, and since $\left|W^{\prime}(\xi)\right| \leq C \xi^{3}+C$, we get

$$
\begin{aligned}
(I I) & \leq \delta \int_{\Omega}(\Delta u)^{2} d x+C \int_{\Omega}|u|^{3} d x+C \\
& \leq \delta \int_{\Omega}(\Delta u)^{2} d x+\delta \int_{\Omega} u^{4} d x+C
\end{aligned}
$$

Putting together our estimates (5.10) for $(I)$ and (5.11) for $(I I)$ together with (5.6), we get

$$
\begin{aligned}
\int_{\Omega}(\Delta u)^{2} d x+\lambda_{0} \int_{\Omega \backslash D}(f-u)^{2} d x & =(I)+(I I) \\
\leq & \delta \int_{\Omega}(\Delta u)^{2} d x+C(\delta) \int_{\Omega} u^{2} d x+C \\
& -(C-\delta) \int_{\Omega} u^{4} d x-C\left(\int_{\Omega} u^{2} d x\right)^{2} .
\end{aligned}
$$


Choosing $\delta>0$ small enough, one gets

$$
\int_{\Omega}(\Delta u)^{2} d x+\lambda_{0} \int_{\Omega \backslash D}(f-u)^{2} d x \leq C(\delta) \int_{\Omega} u^{2} d x-C\left(\int_{\Omega} u^{2} d x\right)^{2}+C .
$$

Let $\xi:=\int_{\Omega} u^{2} d x$. Then the right hand side of $(5.12)$ is $-C \xi^{2}+C(\delta) \xi+C$ for some positive constants $C$ depending on $\delta$. This is a parabola opening downwards, and hence is bounded from above by a constant. That proves the proposition.

5.2. Matching isophotes as $\lambda_{0}$ becomes large. Of interest is what happens to the solution $u(x)$ for the modified Cahn-Hilliard equation when $\lambda_{0}$ is prescribed very large values. Will $u(x)$ correctly match $f(x)$ (the existing image) on the boundary of the inpainting domain? In particular, will the isophote directions be matched? In this section we consider a smooth function $f$ and show that in regions where $f$ changes significantly, the direction of isophotes of the solution will match the direction of isophotes of the prescribed image function. In the next section we extend this result to a binary image with sharp edges. We first establish the following lemma showing that an $H^{2}$ steady state solution is actually in $C^{2, \alpha}$. This results is necessary to establish a pointwise bound for the isophotes on the boundary.

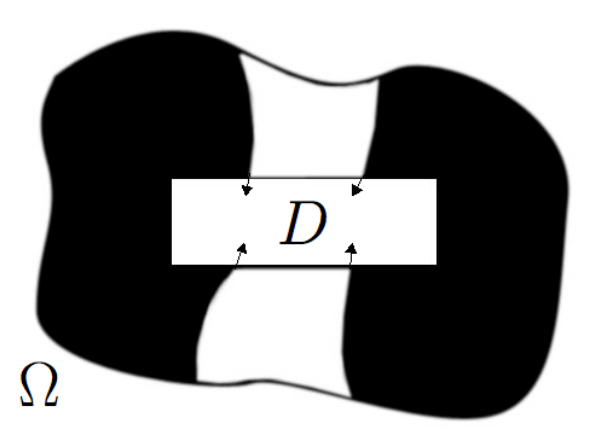

FiG. 5.1. Inpainting problem. The isophote vectors are shown.

Lemma 5.4. Let $\Omega$ have a $C^{2, \alpha}$ boundary and let $u$ be an $H^{2}$ weak solution of the PDE

$$
-\Delta\left(\Delta u-W^{\prime}(u)\right)+\lambda(x)(f-u)=0
$$

where

$$
\lambda(x)=\left\{\begin{array}{cl}
0 & \text { if } x \in D \\
\lambda_{0} & \text { if } x \in \Omega \backslash D
\end{array}\right.
$$

Then $u \in C^{2, \alpha}(\Omega)$.

Proof: By assumption, we have that $\lambda(x)(f-u) \in L^{2}(\Omega)$. Thus

$\Delta\left(\Delta u-W^{\prime}(u)\right) \in L^{2}(\Omega)$ as well. This means that $\Delta u-W^{\prime}(u) \in H^{2}(\Omega)$ [15]. Since $u(x, t)$ is bounded in $H^{2}(\Omega)$ by assumption, it has an a priori pointwise bound, which then implies a pointwise bound on $W^{\prime}(u)$. Sobolev embedding implies that $u \in C^{\alpha}(\Omega)$ and thus $W^{\prime}(u) \in C^{\alpha}(\Omega)$ for all $0 \leq \alpha<1$. This implies that $\Delta u \in C^{\alpha}(\Omega)$, and thus by elliptic regularity [17], $u \in C^{2, \alpha}(\Omega)$. 
Remark: The assumptions on smoothness of the boundary are necessary to invoke the theory of elliptic regularity. In practice for many imaging applications, $\Omega$ is a square with periodic or reflective boundary conditions. In either of those cases, the domain can be viewed as a manifold without boundary and thus the Lemma is applicable. The details of the boundary would only be important for problems where the inpainting region includes some of the boundary of the image domain.

As part of the proof, we first prove the continuity of $\nabla u$ and then show that this leads to matching of the isophote directions in regions where $\nabla f$ is large enough. This is sufficient to show continuity of the direction of edges, since they necessarily imply that $\nabla f$ is large. We now show that in regions where the image intensity changes, the isophote direction of $f$ matches that of $u$ on $\partial D$. This is the main theorem for this section.

TheOREm 5.5. Let $\Omega$ satisfy the same conditions as in Lemma 5.4. Assume that the inpainting region has a piecewise smooth boundary. Consider isophote directions of $f$ in any region where $|\nabla f|>\delta_{0}$. The difference of the isophote vectors, at a smooth part of the boundary of the inpainting region $\partial(D)$, between the steady state solution to the modified Cahn-Hilliard equation $u(x)$, and the known image $f(x)$, can be made arbitrarily small by choosing $\lambda_{0}$ large enough.

Proof: Define $g(x)=(u-f)(x)$. First, we would like to show that $\nabla g(x)$ becomes small pointwise on $\partial(\Omega \backslash D)$ as $\lambda_{0}$ becomes large. From (5.12) we have that

$$
\begin{array}{r}
\int_{\Omega}(\Delta g)^{2} d x \leq C_{1} \\
\int_{\Omega \backslash D}|g(x)|^{2} d x \leq \frac{C_{2}}{\lambda_{0}}
\end{array}
$$

The bounds from 5.13 combined with Sobolev interpolation imply that the $H^{1-\mu}(\Omega \backslash$ $D$ ) norm of $\nabla g$ is small as $\lambda_{0} \rightarrow \infty$, for $0<\mu<\frac{1}{2}$. The restriction map to $\partial D$ (see [16], page 225) implies that $\nabla g$ is small in $H^{1 / 2-\mu}(\partial D)$. Since $L^{2}(\partial D) \subset H^{1 / 2-\mu}(\partial D)$ for $0<\mu<1 / 2$, we have that the $L^{2}$ norm of $\nabla g$ is small on $\partial D$. Continuity of $\nabla g$ implies a pointwise bound on $\nabla g$ on the boundary, in particular we have a constant $\eta(\lambda) \rightarrow 0$ as $\lambda \rightarrow \infty$ such that $\mid \nabla g \|_{\partial D} \leq \eta$. Now we show that this pointwise bound for $\nabla g$ implies a bound for the direction of the isophotes.

Let $\frac{\nabla^{\perp} u}{\left|\nabla^{\perp} u\right|}=\vec{\tau}_{u}$ (this is the isophote vector). We want to show that $\left|\vec{\tau}_{u}-\vec{\tau}_{f}\right|$ becomes small on $\partial(\Omega \backslash D)$ as $\lambda_{0}$ takes increasingly large values. Recall that we are only interested in those portions of $\partial(D)$ where $\nabla^{\perp} f>\delta_{0}$, with $\delta_{0}$ small. Some straightforward algebra shows

$$
\left|\vec{\tau}_{u}-\vec{\tau}_{f}\right| \leq \frac{2|\nabla g|}{\delta_{0}}
$$

Since $\nabla g$ is small for large $\lambda_{0}$, we have the desired result. The case where $\nabla^{\perp} f \leq \delta_{0}$ is not interesting, for in these regions the image is nearly constant and thus does not produce any significant edges. This completes the proof of Theorem 5.5.

6. Matching of isophotes for binary images: continuity of the edge direction. The previous analysis considered the modified Cahn-Hilliard equation (3.1) with $\varepsilon=1$. In real applications involving binary images, we take $\varepsilon$ small as it defines a diffuse interface thickness. Our previous estimates are for smooth functions 
$f$ and in order to apply these ideas, we regularize a binary $f$ at the same scale as the diffuse interface thickness $\varepsilon$. We state this problem as follows: consider a binary image function $f$ taking values 0 and 1 . Assume a smooth boundary between regions where $f=0$ and $f=1$. Using a mollifier, construct $f_{\varepsilon}=J_{\varepsilon} f(x)$. A simple way to do this is to solve the heat equation on $\Omega$, with Neumann boundary conditions and initial condition $f$, until time $t=\varepsilon^{2}$. This gives a smooth approximation of $f$ in which the edges of the images are smoothed over a scale of length $\varepsilon$.

We now solve the inpainting problem by evolving the time dependent equation

$$
u_{t}=-\Delta\left(\varepsilon \Delta u-\frac{1}{\varepsilon} W^{\prime}(u)\right)+\lambda(x)\left(f_{\varepsilon}-u\right) .
$$

where

$$
\lambda(x)=\left\{\begin{array}{cl}
0 & \text { if } x \in D \\
\lambda_{0} & \text { if } x \in \Omega \backslash D
\end{array}\right.
$$

Global existence of a weak solution of the above problem follows from the arguments in section 4 . We now consider the steady state problem

$$
-\Delta\left(\varepsilon \Delta u-\frac{1}{\varepsilon} W^{\prime}(u)\right)+\lambda(x)\left(f_{\varepsilon}-u\right)=0
$$

where

$$
\lambda(x)=\left\{\begin{array}{cl}
0 & \text { if } x \in D \\
\lambda_{0} & \text { if } x \in \Omega \backslash D
\end{array}\right.
$$

and follow the arguments of the preceding section to show that for sufficiently large $\lambda_{0}$, the steady state solution above has edges matching those of the original image $f$. To do this, we show that for a fixed $\varepsilon, \lambda$ can be chosen large enough so that the isophote directions are nearly parallel on the boundary of $D$.

We consider the analogous estimates to (5.4) for the case $\varepsilon \neq 1$. Take the inner produce of the steady state equation with $(u-f)$. We obtain

$$
\varepsilon \int_{\Omega}(\Delta u)^{2} d x+\int_{\Omega \backslash D} \lambda_{0}(f-u)^{2}=\frac{1}{\varepsilon} \int_{\Omega} W^{\prime}(u) \Delta u d x+\int_{\Omega}\left(\varepsilon \Delta u-\frac{1}{\varepsilon} W^{\prime}(u)\right) \Delta f d x .
$$

This in turn leads to the estimate

$$
\begin{aligned}
\varepsilon \int_{\Omega}(\Delta u)^{2} d x+\lambda_{0} \int_{\Omega \backslash D}(f-u)^{2} d x= & (I)+(I I) \\
\leq & \left(\varepsilon+\frac{1}{\varepsilon}\right) \delta \int_{\Omega}(\Delta u)^{2} d x+\frac{1}{\varepsilon} C(\delta) \int_{\Omega} u^{2} d x+C\left(\frac{1}{\delta}, \varepsilon+\frac{1}{\varepsilon}\right) \\
& -\frac{1}{\varepsilon}(C-\delta) \int_{\Omega} u^{4} d x-\frac{1}{\varepsilon} C\left(\int_{\Omega} u^{2} d x\right)^{2} .
\end{aligned}
$$

Now letting $\delta$ become very small, as $\varepsilon$ is fixed, we have that

$\varepsilon \int_{\Omega}(\Delta u)^{2} d x+\lambda_{0} \int_{\Omega \backslash D}(f-u)^{2} d x \leq-\frac{1}{\varepsilon} C\left(\int_{\Omega} u^{2} d x\right)^{2}+\frac{1}{\varepsilon} C \int_{\Omega} u^{2} d x+C\left(\varepsilon+\frac{1}{\varepsilon}\right)$. 
Once again, the right hand side of the inequality is a parabola that opens downward. Thus:

$$
\varepsilon \int_{\Omega}(\Delta u)^{2} d x+\lambda_{0} \int_{\Omega \backslash D}(f-u)^{2} d x \leq C\left(\varepsilon+\frac{1}{\varepsilon}\right),
$$

from which we obtain the bounds

$$
\begin{aligned}
& \int_{\Omega}(\Delta u)^{2} d x \leq C\left(\left(\varepsilon+\frac{1}{\varepsilon}\right) \frac{1}{\varepsilon}\right), \\
& \int_{\Omega \backslash D}(f-u)^{2} d x \leq \frac{C\left(\varepsilon+\frac{1}{\varepsilon}\right)}{\lambda_{0}} .
\end{aligned}
$$

This shows the full relationship between $\lambda_{0}$ and $\varepsilon$. It is important to notice that the constants $C\left(\frac{1}{\varepsilon^{2}}\right)$ and $C\left(\varepsilon+\frac{1}{\varepsilon}\right)$ become very large as $\varepsilon$ becomes small. Thus, for small $\varepsilon, \lambda_{0}$ must be chosen very large to guarantee continuity of edges using these estimates.

Using the results of section 5.2 , we have that $\nabla\left(u-f_{\varepsilon}\right)(x) \rightarrow 0$ on $\partial D$ as $\lambda_{0} \rightarrow \infty$. Consider now the part of $\partial D$ where $\left|\nabla f_{\varepsilon}\right|>\delta_{0}$. Since the original $f$ is binary, this region corresponds to a narrow band around the edges of the original $f$. Following the ideas in section 5.2, we see that the isophote vectors $\left|\vec{\tau}_{u}-\vec{\tau}_{J_{\varepsilon} f}\right| \rightarrow 0$ in this narrow band which defines the diffuse interface between regions where $f=0$ and $f=1$. Putting this all together, we consider an original $f$ taking values 0 and 1 with smooth boundary between the two phases. Assume that $\varepsilon$ is small enough so that $\tau_{f_{\varepsilon}}$ is almost parallel to the edge direction of the original binary $f$ in the region where $\left|\nabla f_{\varepsilon}\right|>\delta_{0}$. The solution $u$ of the steady state diffuse interface problem will have edges that line up with those of $f_{\varepsilon}$ and thus with the original binary $f$, provided that $\lambda_{0}$ is large enough (depending on our choice of $\varepsilon$ ).

In the above discussion, we implicitly assume a 'separation of scales' in the solution $u$. If we assume $f$ is a binary image with order one features and curvature of edges, then the regularized $f_{\epsilon}$ is guaranteed to have a separation of scales, meaning that it consists of regions separated by diffuse interfaces where there is steep variation (on a spatial scale of order $\epsilon$ ) normal to the diffuse interface and very mild variation tangent to the interface direction. If the solution $u_{\epsilon}$ has the same separation of scales as the regularized data $f_{\epsilon}$, then the result will be a matching of edges between the data and the solution, for large $\lambda$. Note that the estimates derived above require $\lambda$ to possibly be very large, depending badly on $\epsilon$. In our analysis here we do not prove that a separation of scales occurs for the solution $u$ in the inpainting region, however the computational results of the following sections illustrate this to be the case. Such a result is beyond the scope of this paper but would be interesting to examine in its own right. The original asymptotic analysis for separation of scales for the plain Cahn-Hilliard equation was carried out by Pego [28]. The analysis is local and thus should hold in the interior of the inpainting region where the fidelity term is zero. Our simulations are observed to follow the same scaling as the original Cahn-Hilliard equation, in the inpainting region. In Section 8, we present numerical results illustrate that separation of scales for $u$ and thus continuation of edge direction does occur for this model. See in particular Figures 8.2-8.4.

In addition to having two spatial scales, the original Cahn-Hilliard asymptotics shows separation of time scales. There is a short time scale on which phase separation occurs, and a longer time scale (related to $\epsilon$ ) on which the diffuse interface boundary moves. These same time scales are present in our modified equation. In the analysis 
above we consider the steady state problem. In the numerical examples, $\lambda$ is chosen large enough so that the 'fidelity timescale' is short compared to the other timescales in the problem associated with the regular Cahn-Hilliard dynamics. The timescale associated with the motion of the interface must be addressed when designing fast algorithms for binary inpainting. In Section 8 we make use of this separation of timescales to design a two-step algorithm in which reconnection of shapes is first performed with a large $\epsilon$, thereby decreasing the timescale of reconnection. Then we suddenly decrease $\epsilon$ to sharpen the interface, which also happens on a short timescale as this dynamics is associated with phase separation rather than interface motion. The need for a two-step method is further explained by the stripe reconnection examples in the following section. There we compute bifurcation diagrams for steady states associated with a single stripe reconnection. For a large gap width, the connected stripe solution is a separate branch from the branch of solutions that contains the stable solution for large $\epsilon$. We explain this in more detail in the following section.

7. Bifurcations of the Modified Cahn-Hilliard Equation. A natural question to ask is whether the steady state solution is unique. Here we show by numerical examples that multiple solutions exist and can be understood through a bifurcation analysis. We conducted tests of the modified Cahn-Hilliard equation

$$
u_{t}=-\Delta\left(2 \varepsilon \Delta u-\frac{1}{\varepsilon} W^{\prime}(u)\right)+\lambda_{0}(f-u) \chi_{\Omega \backslash D}
$$

on a simple stripe geometry. The numerical scheme used is based on convexity splitting and is discussed in detail in $[6,37]$. We note that while the theory presented in this manuscript assumes Neumann boundary conditions, the computations here are done with periodic boundary conditions. However one can think of Neumann BC as a restriction to the periodic problem with symmetry, simply by reflecting across domain edges in a square domain. Figure 7.1 shows an example where the inpainting domain $D$ is the grey region in frame (a). Different initial conditions for the dynamic problem (7.1) yield different steady state solutions as shown in frames (b) and (c). In both examples we take the same initial condition $u_{0}=f$ in $\Omega \backslash D$ and $u_{0}=0$ in $D$. However, in the case of (b) we start with a large $\varepsilon=0.8$, run the solution to steady state, and use this as a new initial condition for a smaller value of $\varepsilon$. In the case (c) we perform a single simulation with fixed $\varepsilon$ small (0.01), starting from $u_{0}$ given above. We can not take $\epsilon$ much smaller without having to increase the resolution of the grid. In practice, the Cahn-Hilliard dynamics is reasonably well-captured with a few grid points resolving the diffuse interface scale $\epsilon[19,37]$. A much coarser grid can result in numerical pinning of the interface.

The bifurcation diagrams below in figure 7.2 show how the steady states, for the modified Cahn-Hilliard equation, change in response to changes in the value of $\varepsilon$ (reference equation 7.1). We consider the stripe problem as above, for different gap widths of 30,45 , and 80 . We choose an amplitude for the bifurcation diagrams of the value of the steady state solution at the center of the inpainting domain $D$. This is a useful measure in that a completed stripe will have an amplitude close to one, whereas a broken stripe will have an amplitude close to zero. Intermediate values are observed for steady states in which the diffuse interface scale $\varepsilon$ is comparable to the feature size in the problem.

Note that in all figures, only stable steady states are shown as we use the time dependent PDE to obtain the steady states. These figures suggest the presence of an incomplete pitchfork bifurcation. A diagram for this type of bifurcation is shown in 

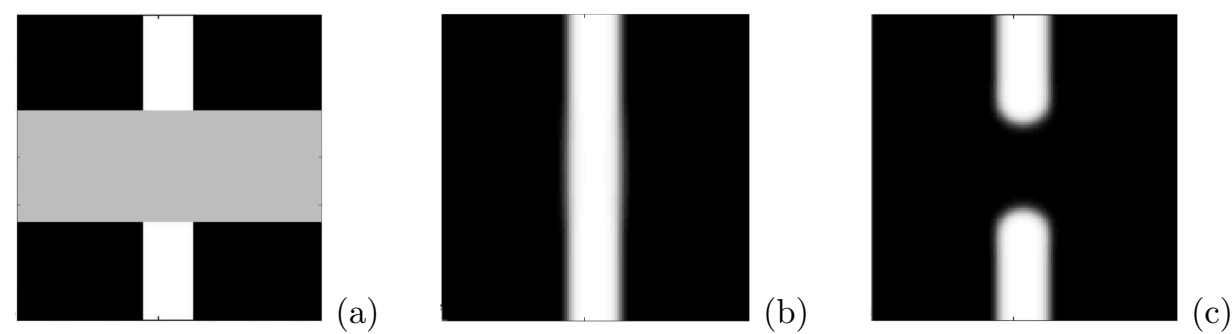

FIG. 7.1. (a) Grey portion denotes the inpainting region $D$ while the black and white portion denote that background image $f$. (b) steady state solution of (7.1) showing a completed stripe, (c)steady state solution of (7.1) showing a broken stripe. In both cases $\varepsilon=0.01$. In all cases we choose a square of $128 \times 128$ grid points, with the inpainting region having a gap of width 40 grid points. The grid spacing is $\Delta x=0.01$.

figure $7.3[34]$ in which stable branches appear as solid lines and unstable branches appear as dotted lines. Frame (b) shows the classical complete pitchfork bifurcation. Changes in parameters, including, but not limited to symmetry breaking, can cause a section of the pitchfork to break off into a stable/unstable pair of solutions, as shown in frames (a) and (c). It is interesting to compare the diagram in figure 7.3 with the numerically obtained diagrams in figure 7.2. In frame (a) there is an unbroken stable branch connecting the single large $\varepsilon$ solution to the connected stripe solution for small $\varepsilon$. In contrast, in frames (b) and (c), the unbroken stable branch connects the single large $\varepsilon$ solution to the broken stripe solution for small $\varepsilon$. In these cases the connected stripe solution appears as an isolated branch. We conjecture that the isolated branch flips over to an unstable branch of steady state solutions in all three cases. In frame (a) it is the broken stripe solution that forms an isolated branch.

The bifurcation diagrams above suggest that different approaches will be necessary to obtain the completed stripe solution, depending on the gap width. For the small gap case, one can simply compute the steady state at large $\varepsilon$ and continuously shrink $\varepsilon$, following the stable branch, to the desired small $\varepsilon$. However this approach will clearly not work for the larger gap widths. Instead, we find that a two-scale approach empirically works well to obtain the continued stripe solution. We choose, at the outset, a value of $\varepsilon$ on the order of the maximum gap size. For example, choosing $\varepsilon=.8$ as a starting value, we find a unique steady state solution for a very diffuse scale. Instead of continuously lowering $\varepsilon$, we abruptly change to the desired small scale value and find empirically that the continued stripe solution emerges from the dynamics. In summary, our algorithm for finding the completed stripe solutions is as follows:

1. Choose an initial value of $\varepsilon$ nearly equal to the numerical maximum gap spacing (above $\varepsilon=.8$ was used). Set $\Delta t=1$, with $\Delta x=\Delta y=.01$. The image size is taken to be $128 \times 128$ grid points, each of size $\Delta x$.

2. Run the modified Cahn-Hilliard equation to near steady state (300 iterations) for this value of $\varepsilon$.

3. At 300 iterations, multiply the near steady state solution $u(x, y)$ by a factor more than 1 so that $\max [u(x, y)]=1.0$.

4. Still at 300 iterations, switch $\varepsilon$ to a value of .01 (approximately the numerical grid spacing value $\Delta x$ ).

In practice, to obtain the steady state requires less than 30 seconds on a Pentium 4 processor. Figure 7.4 (a), (b), (c), shows an example of such a calculation. 


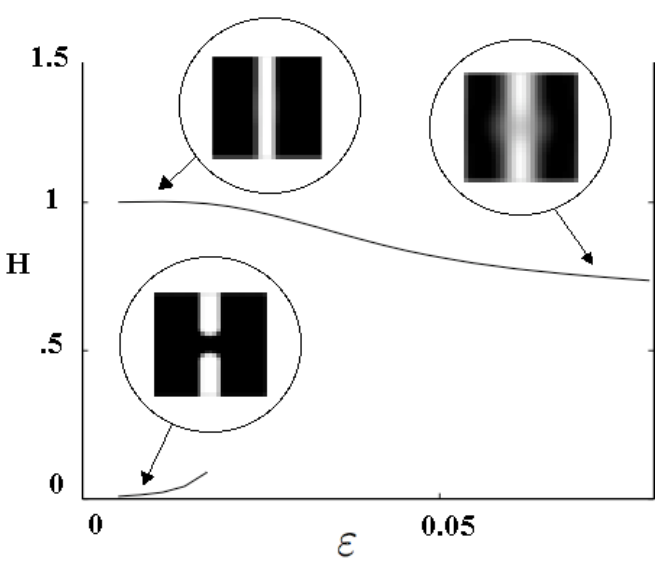

(a)

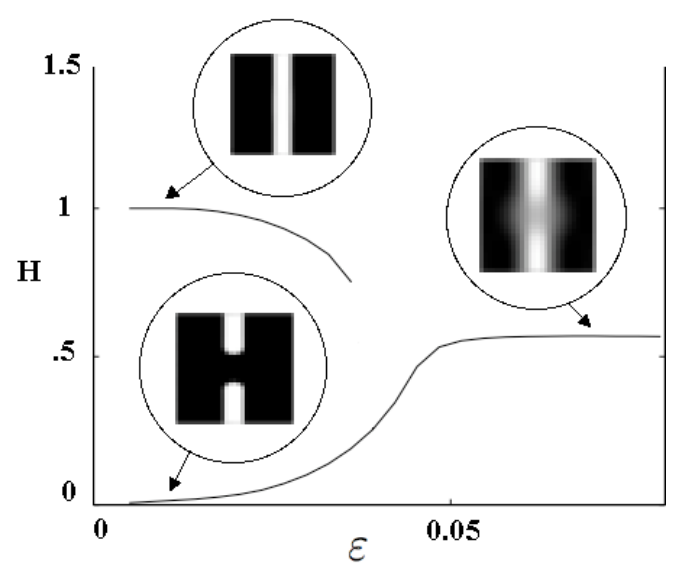

(b)

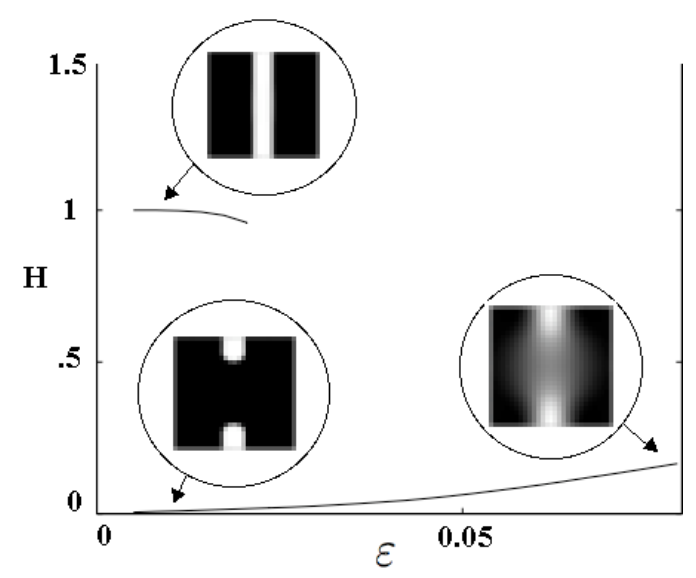

(c)

FIG. 7.2. Bifurcation diagrams for, (a) gap of 30, (b) gap of 45, (c) gap of 80. The yaxis shows the steady state height of the midpoint of the stripe, while the $x$-axis is the epsilon value. The steady states are shown visually in thumbnails, with an arrow pointing to their positions on the respective bifurcation diagrams. Only stable steady states are shown.

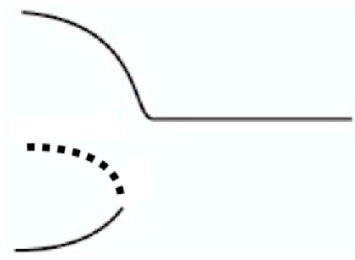

(a)

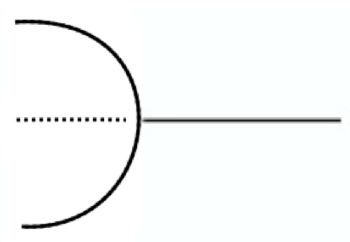

(b)

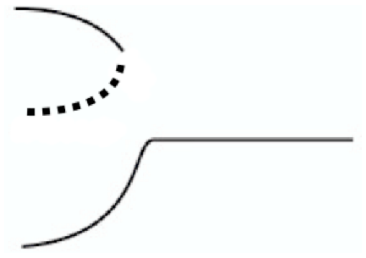

(c)

FIG. 7.3. Bifurcation diagrams relating to the phenomena shown in figure 7.2. (a) An incomplete pitchfork bifurcation. (b) A symmetric pitchfork bifurcation. (c) Another incomplete pitchfork bifurcation. 


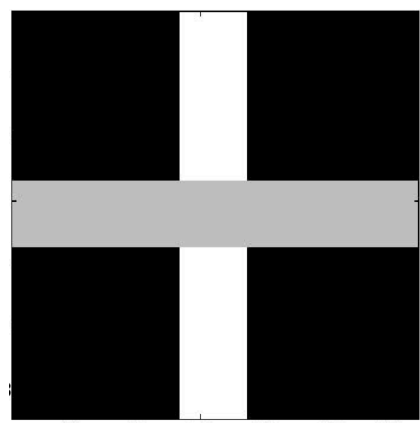

(a)

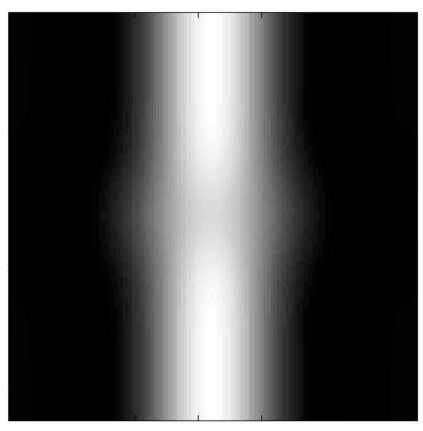

(b)

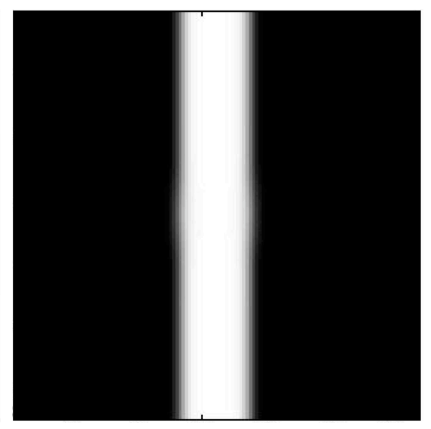

(c)

FIG. 7.4. Disconnected stripe with a gap of 20, at (a) $t=0$, (b) $t=299$, after steady state had been reached for $\varepsilon=.8$, (c) $t=500$ (produced by switching to $\varepsilon=.01$ at $t=300$ )

8. Numerical Examples. In a previous paper (see [6]) introducing this method, we showed some numerical examples that illustrate the performance of a convexity splitting scheme for this problem, as compared to other recent PDE-based inpainting methods. Here we provide some examples illustrating the role of $\varepsilon$ and $\lambda_{0}$. The modified Cahn-Hilliard equation was tested against a variety of elementary inpainting geometries, as well as several more complex cases. Below and on the next pages, we repeat some examples from the previous paper, and as well present a new example showing binary inpainting of text.

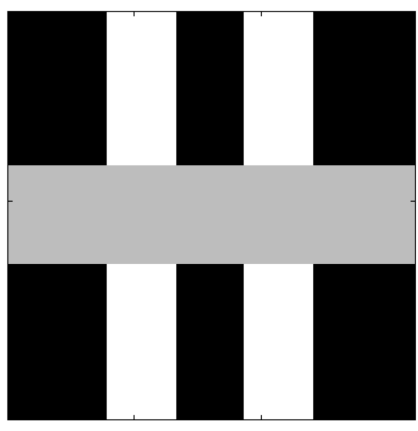

(a)

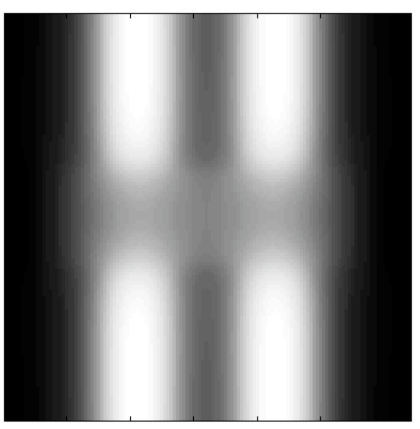

(b)

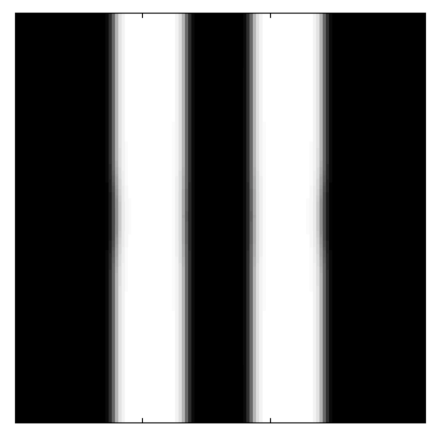

(c)

FIG. 8.1. (a) Initial data (inpainting region in grey). (b) Intermediate state at $t=50$. (c) steady state at $t=700$. (Gap distance is 30 grid points, Image domain is $128 \times 128$ ).

8.1. Inpainting of a double stripe. Above in figure 8.1, we see the two-step process at work to inpaint two stripes. The grey region in figure 8.1(a) denotes the inpainting region. We begin running the modified Cahn-Hilliard equation with a large value of $\varepsilon(=.8)$, and at $t=50$ we come close to a steady state, shown in figure 8.1(b). We then switch to a small value of $\varepsilon(=.01)$, using the result from figure 8.1(b) as initial data. The final result at $t=700$, is shown in figure 8.1(c). In this test, $\Delta t=1$, $\lambda=50,000, C_{1}=300$, and $C_{2}=150,000$.

8.2. Inpainting of a cross. In figure 8.2(a), the grey region denotes the "gap", or region to be inpainted. As with the stripes, the modified Cahn-Hilliard equation is run to steady state for a large value of $\varepsilon(=.8)$, resulting in figure 8.2(b) at $t=300$. 


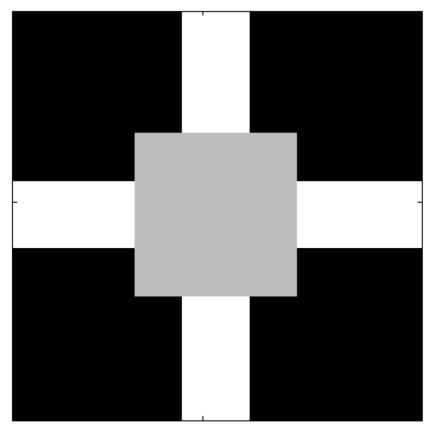

(a)

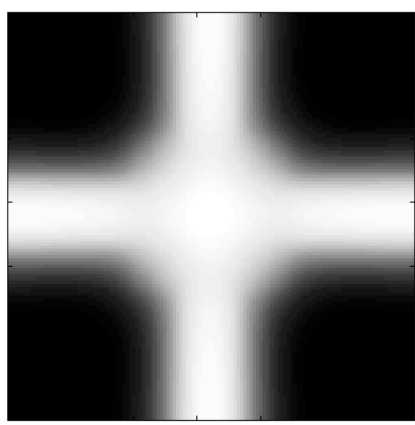

(b)

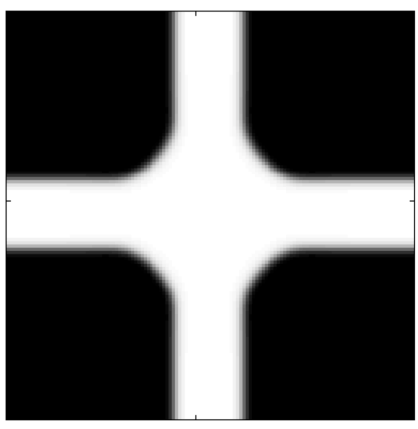

(c)

FIG. 8.2. (a) Initial data of cross (inpainting region in grey). (b) Intermediate state at $t=300$. (c) Steady state at $t=1000$. (Image domain is $128 \times 128$, stripe width is 20 grid points, initial gap distance is 50 grid points).

This data is then used as initial data for the modified Cahn-Hilliard equation with $\varepsilon(=.01)$ set to a small value. The final result is a completed cross at $t=1000$. The parameters are $\Delta t=1, \lambda=100,000, C_{1}=300$, and $C_{2}=3 \lambda$.

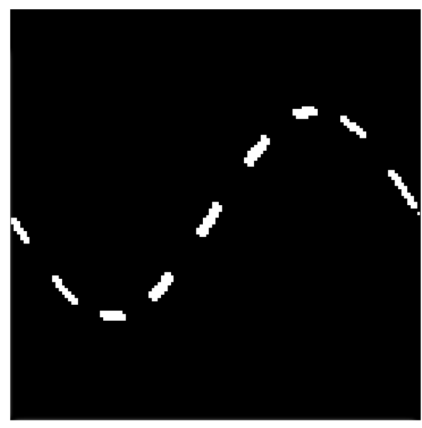

(a)

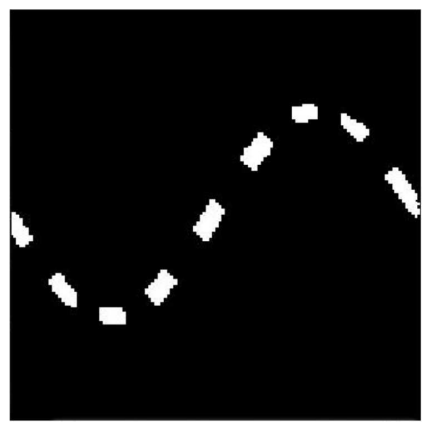

(b)
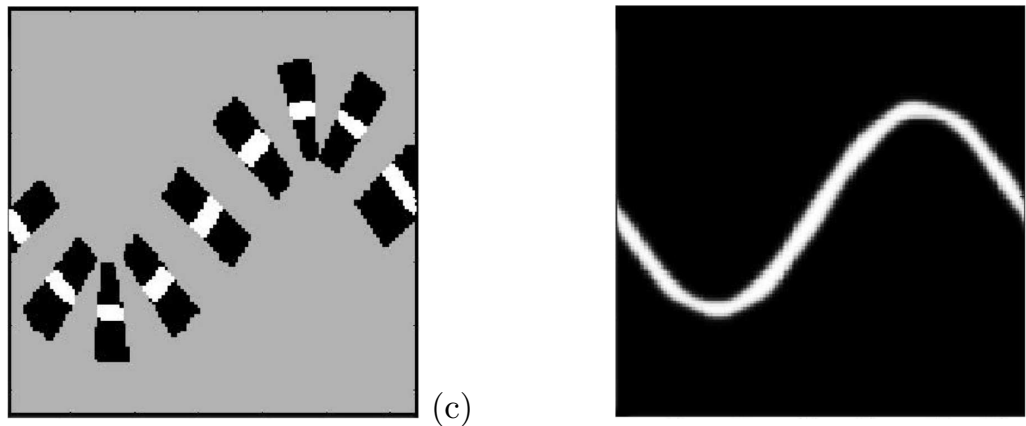

(d)

FIG. 8.3. Inpainting a sine wave. (Image domain is $128 \times 128$ ).

8.3. Inpainting of a Sine Wave. Figure 8.3 shows how the modified CahnHilliard equation may be applied to the inpainting of simple road-like structures. In figure 8.3(a), an incomplete sine wave is shown. In figure $8.3(\mathrm{~b})$, the sine wave is artificially "fattened" by expanding each white point's area radially by a factor of 3 . This is done in order to give the modified Cahn-Hilliard equation sufficient boundary conditions to do effective inpainting. 
In figure 8.3(c), the grey area represents the inpainting region. The remaining white and black portions of the image are thus outside the inpainting region, and essentially held fixed in place by the fidelity term of the modified Cahn-Hilliard equation (7.1). The two step method was then used to inpaint the sine wave. Figure 8.3(d) shows the finished result.

The initial value of $\varepsilon$ was taken to be .8 , and then at $t=200$ this was switched to a value of $\varepsilon=.01$. The final inpainting result was taken at $t=4000$ (which corresponds to a time of 24 seconds real processing time). The parameters were set as: $\Delta t=1, C_{1}=300, \lambda=100,000$, and $C_{2}=3 \lambda$.

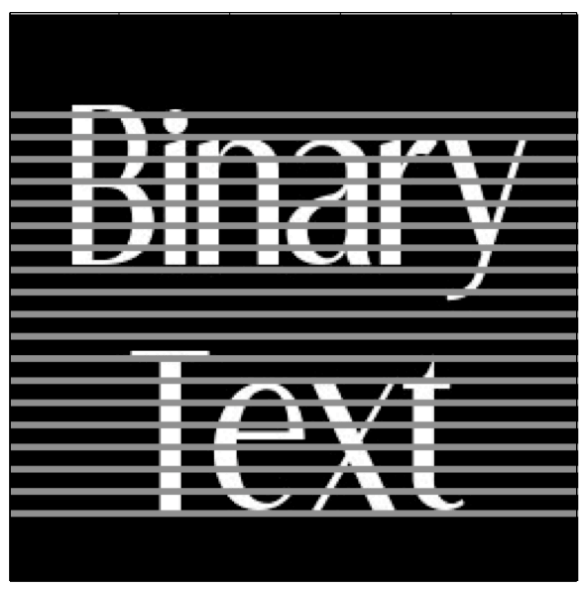

(a)

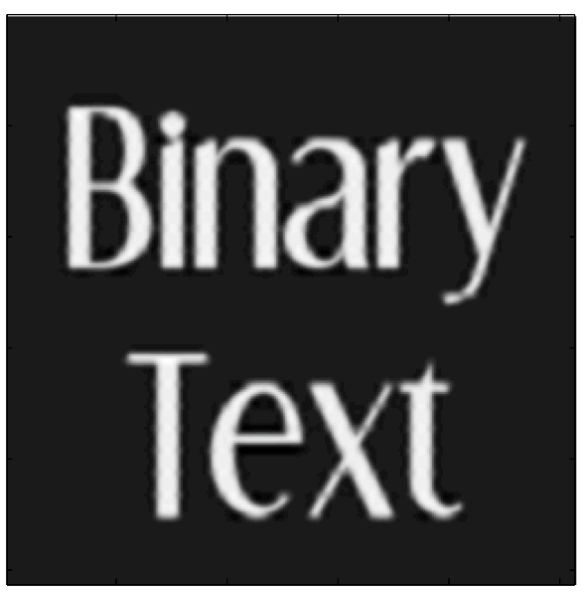

(b)

FiG. 8.4. Recovering obscured text. (Image domain is $256 \times 256$ ).

8.4. Inpainting of obscured text. Figure 8.4 shows how the modified CahnHilliard equation can be used to recover obscured text. Figure 8.4(a) shows the text obscured by lines. This is a common problem for Optical Character Recognition (OCR) algorithms, with regard to text written on lined paper. Figure 8.4(b) shows the result after processing by the modified Cahn-Hilliard equation.

The parameters were set as $\Delta t=1, \lambda=100,000,000, C_{1}=10,000, C_{2}=3 \lambda$, $\varepsilon=.008$, and were kept constant during this particular test. The test was completed at time $t=800$, which corresponds to 2 minutes of processing time.

9. Conclusions. This paper considers the method for binary inpainting recently introduced by the authors in [6]. This work introduces a relatively simple fourth order PDE for this task, rather than a more complex gradient flow to minimize a curvature functional. This simpler model, based on the Cahn-Hilliard has many of the desirable properties of earlier models, in particular the ability to match color and direction of edges, without requiring the computational complexity of those methods. In this manuscript we prove that for the proposed $\mathrm{CH}$ model, steady state solutions do indeed inherit the observed boundary conditions in the large fidelity limit.

Empirically, we find that one can perform inpainting across larger regions by considering a two-step method. The inpainting is done first with a larger $\varepsilon$, which 
results in topological reconnection of shapes with edges smeared by diffusion. The second step then uses the results of the first step and continues with a much smaller value of $\varepsilon$ in order to sharpen the edge after reconnection.

In practice such a two-stage process can result in inpainting of a stripe across a region that is over ten times the width of the stripe, without any a priori knowledge of the location of the stripe. This two-step method is an empirically observed phenomenon which we understand more fully by considering the problem of bifurcations due to the presence of multiple stable steady state solutions to the same inpainting problem. Through a numerical search, we present evidence that the dynamical problem undergoes an incomplete pitchfork bifurcation, from a single stable steady state solution at large $\varepsilon$ to two co-existing stable steady states at small $\varepsilon$, taking the form of a broken stripe with rounded ends, and a completed stripe. One of these two solutions is connected to the large $\varepsilon$ by a continuous branch in the bifurcation diagram. For large gap widths, it is the broken stripe, thereby requiring a somewhat more complex process to find the basin of attraction of the completed stripe. Fortunately this is not so difficult using the two-step process described above.

We complete the paper by showing some computational examples. This scheme was shown in [6] to outperform in speed other variationally derived inpainting methods by a significant amount (typically an order of magnitude or more). Thus it would be interesting to consider methods related to this one for more complex inpainting problems such as greyscale, color, and nonlocal filling-in.

Finally we mention that while this manuscript focuses on PDE-based methods for filling in, there are many other approaches in the literature. Perhaps the closest to what is considered here are those based on spline continuation of the edges [32, 33].

\section{REFERENCES}

[1] Nicholas D. Alikakos, Peter W. Bates, and Xinfu Chen. Convergence of the Cahn-Hilliard equation to the Hele-Shaw model. Arch. Rational Mech. Anal., 128(2):165-205, 1994.

[2] L. Ambrosio and V. M. Tortorelli. Approximation of functionals depending on jumps by elliptic functionals via gamma convergence. Communications on Pure and Applied Mathematics, 43:999-1036, 1990.

[3] P. Bates and P. Fife. The dynamics of nucleation for the Cahn-Hilliard equation. SIAM J. Appl. Math, 53(4):553-582, 1993.

[4] M. Bertalmio, A. Bertozzi, and G. Sapiro. Navier-Stokes, fluid dynamics, and image and video inpainting. IEEE Computer Vision and Pattern Recognition (CVPR), Hawaii, 1:I355I362, December 2001.

[5] M. Bertalmio, G. Sapiro, V. Caselles, and C. Ballester. Image inpainting. In Kurt Akeley, editor, Siggraph 2000, Computer Graphics Proceedings, pages 417-424. ACM Press / ACM SIGGRAPH / Addison Wesley Longman, 2000.

[6] A. Bertozzi, S. Esedoglu, and A. Gillette. Inpainting of binary images using the Cahn-Hilliard equation. IEEE Trans. Image Proc., 2006. to appear.

[7] C. Braverman. Photoshop retouching handbook. IDG Books Worldwide, 1998.

$[8]$ T. Chan, J. Shen, and S. Kang. Euler's elastica and curvature-based image inpainting. SIAM Journal on Applied Mathematics, 63(2):564-592, 2002.

[9] T. F. Chan and J. Shen. Mathematical models of local non-texture inpaintings. SIAM Journal on Applied Mathematics, 62(3):1019-1043, 2001.

[10] G. Dal Maso. An introduction to Gamma convergence. Progress in nonlinear differential equations and their applications. Birkhauser Boston, Inc., Boston, MA, 1993.

[11] Italo Capuzzo Dolcetta, Stefano Finzi Vita, and Riccardo March. Area-preserving curveshortening flows: from phase separation to image processing. Interfaces and Free Boundaries, 4(4):325-343, 2002.

[12] G. Emile-Male. The restorer's handbook of easel painting. Van Nostrand Reinold, New York, 1976. 
[13] S. Esedoglu and R. March. Segmentation with depth but without detecting junctions. Journal of Mathematical Imaging and Vision, 18:7-15, 2003.

[14] S. Esedoglu and J. Shen. Digital inpainting based on the Mumford-Shah-Euler image model. European Journal of Applied Mathematics, 13:353-370, 2002.

[15] Lawrence C. Evans. Partial differential equations. American Mathematical Society, Providence, RI, 1998.

[16] Gerald B. Folland. Introduction to partial differential equations. Princeton University Press, Princeton, NJ, second edition, 1995.

[17] D. Gilbarg and N. S. Trudinger. Elliptic Partial Differential Equations of Second Order. Springer, 1998. Revised Third Printing.

[18] E. De Giorgi. Some remarks on gamma-convergence and least squares methods. In G. Dal Maso and G. F Dell'Antonio, editors, Composite Media and Homogenization Theory, pages 135142. Birkhauser, 1991.

[19] K. Glasner. A diffuse interface approach to Hele-Shaw flow. Nonlinearity, 16:49-66, 2003.

[20] D. King. The Commissar Vanishes. Henry Holt and Company, 1997.

[21] A. C. Kokaram. Motion Picture Restoration: Digital Algorithms for Artefact Suppression in Degraded Motion Picture Film and Video. Springer Verlag, 1998.

[22] R. March and M. Dozio. A variational method for the recovery of smooth boundaries. Image and Vision Computing, 15:705-712, 1997.

[23] S. Masnou. Filtrage et Desocclusion d'Images par Méthodes d'Ensembles de Niveau. PhD thesis, Univ. Paris-Dauphine, 1998.

[24] S. Masnou and J. M. Morel. Level-lines based disocclusion. 5th IEEE International Conference on Image Processing, 1998.

[25] L. Modica and S. Mortola. Un esempio di gamma-convergenza. Boll. Un. Mat. Ital. B (5), 14(1):285-299, 1977.

[26] M. Nitzberg, D. Mumford, and T. Shiota. Filtering, Segmentation, and Depth. Number 662 in Lecture Notes in Computer Science. Springer-Verlag, 1993.

[27] A. Novick-Cohen and L. A. Segal. Nonlinear aspects of the Cahn-Hilliard equation. Physica D, 10:277-298, 1984.

[28] R. Pego. Front migration in the nonlinear Cahn-Hilliard equation. Proc. Roy. Soc. London A, 422:261-278, 1989.

[29] P. Perona and J. Malik. Scale-space and edge detection using anisotropic diffusion. IEEE Transactions on Pattern Analysis and Machine Intelligence, 12:629-639, 1990.

[30] M. Roger and R. Schatzle. On a modified conjecture of De Giorgi. Mathematische Zeitschrift, 2006. online first at http://www.springerlink.com/content/225h496348761510/.

[31] L. Rudin, S. Osher, and E. Fatemi. Nonlinear total variation based noise removal algorithms. Physica D, 60:259-268, 1992.

[32] Guido Schuster, Xiaohuan Li, and Aggelos K. Katsaggelos. Spline-based boundary loss concealment. Proceedings of the IEEE International Conference on Image Processing, 2003.

[33] Luis Ducla Soares and Fenando Pereira. Spatial shape error concealment for object-based image and video coding. IEEE Trans. Image Proc., 13(4), 2004.

[34] Steven H. Strogatz. Nonlinear Dynamics and Chaos. Addison-Wesley, 1994.

[35] J. E. Taylor and J. W. Cahn. Linking anisotropic sharp and diffuse surface motion laws via gradient flows. J. Stat. Phys., 77:183-197, 1994.

[36] Roger Temam. Infinite-Dimensional Dynamical Systems in Mechanics and Physics. SpringerVerlag, New York, New York, second edition, 1997.

[37] Benjamin P. Vollmayr-Lee and Andrew D. Rutenberg. Fast and accurate coarsening simulation with an unconditionally stable time step. Phys. Rev. E, 68:66703, 2003. 